\title{
ERROR ESTIMATES FOR DIFFERENTIAL DIFFERENCE SCHEMES TO PSEUDO-PARABOLIC INITIAL-BOUNDARY VALUE PROBLEM WITH DELAY
}

\author{
Pinar Okcu and Gabil M. Amiraliyev \\ Department of Mathematics, Sinop University, 57000, Sinop, Turkey \\ okcupinar@gmail.com
}

\begin{abstract}
We consider the one dimensional initial-boundary Sobolev problem with delay. For solving this problem numerically, we construct fourth order differentialdifference scheme and obtain the error estimate for its solution. Further we use the appropriate Runge-Kutta method for the realization of our differential-difference problem.
\end{abstract}

Key Words- Sobolev Problem, Delay Difference Scheme, Error Estimate

\section{INTRODUCTION}

We consider the initial-boundary value problem for pseudo-parabolic differential equation with delay in the domain $\bar{Q}=\bar{\Omega} \times[0, T] ; \bar{\Omega}=[0, l], Q=\Omega \times(0, T], \Omega=(0, l)$

$$
\begin{aligned}
& \frac{\partial u(x, t)}{\partial t}-a(t) \frac{\partial^{3} u(x, t)}{\partial t \partial x^{2}}=b(t) \frac{\partial^{2} u(x, t)}{\partial x^{2}}+c(t) u(x, t)+d(t) u(x, t-r)+f(x, t),(x, t) \in Q, \\
& u(x, t)=\varphi(x, t),(x, t) \in \bar{\Omega} \times[-r, 0], \\
& u(0, t)=u(l, t)=0, t \in(0, T],
\end{aligned}
$$

where $a \geq \alpha>0, b, c, d, f$ and $\varphi$ are sufficiently smooth functions satisfying certain regularity conditions to be specified, $r>0$ represents the delay parameter.

Equations of this type arise in many areas of mechanics and physics. They are used to study heat conduction [7], homogeneous fluid flow in fissured rocks [5], shear in second order fluids [12,19] and other physical models. The important characteristic of these models is that they express the conservation of a certain quantity (mass, momentum, heat, etc.) in any sub-domain. For a discussion of existence and uniqueness results of pseudo-parabolic equations see $[6,8,13,18]$. Various finite difference schemes have been constructed to treat such problems [1-4] For example in [10] two difference approximation schemes to a nonlinear pseudo-parabolic equation are developed. Each of these schemes possesses a unique solution which can be obtained by an iterative procedure. Further in [17] two difference streamline diffusion schemes for solving linear Sobolev equations with convection-dominated term are given. We can see other numerical methods of this type of equations in $[11,15]$ (see also the references cited in them). In [9] a Crank-Nicolson-Galerkin approximation with extrapolated coefficients is presented for three cases for the nonlinear Sobolev equation along with a conjugate 
gradient iterative procedure which can be used efficiently to solve the different linear systems of algebraic equations arising at each step from the Galerkin method. In [20] the author study a finite volume element approximation of pseudo-parabolic equations in three spatial dimensions.

In this study, we use the method of lines for the discretization in space variable for the problem (1.1)-(1.3). The method of lines is a general technique for solving partial differential equations by typically using finite difference relationships for the spatial derivatives or the time derivative. Our aim is to get a fourth order accurate differential-difference scheme and to establish the error estimate for its solution.

\section{CONSTRUCTION OF THE SCHEME}

On the $\bar{\Omega}$, we introduce the uniform mesh

$\omega_{h}=\left\{x_{i}=i h, i=1,2, \ldots, N-1, h=l / N\right\}$

and denote

$$
g_{\bar{x} x, i}=\frac{g_{i+1}-2 g_{i}+g_{i-1}}{h^{2}}
$$

for any mesh function $g_{i}$.

To construct the difference scheme, we will use the following relation which is valid for any $g(x) \in C^{6}\left[x_{i-1}, x_{i+1}\right]$

$\frac{1}{12}\left[g^{\prime \prime}\left(x_{i+1}\right)+10 g^{\prime \prime}\left(x_{i}\right)+g^{\prime \prime}\left(x_{i-1}\right)\right]=g_{\bar{x} x, i}+\bar{R}_{i}$,

where

$$
\begin{aligned}
& \bar{R}_{i}=h^{-1} \int_{x_{i-1}}^{x_{i+1}} \frac{\partial^{6} g}{\partial x^{6}}\left(\xi_{i}\right) \Lambda(\xi) d \xi \\
& \Lambda(\xi)=\left\{\begin{array}{l}
\frac{h}{72}\left(x_{i+1}-\xi\right)^{3}-\frac{h^{-1}}{120}\left(x_{i+1}-\xi\right)^{5}, \xi>x_{i} \\
\frac{h}{72}\left(\xi-x_{i-1}\right)^{3}-\frac{h^{-1}}{120}\left(\xi-x_{i-1}\right)^{5}, \xi<x_{i}
\end{array}\right.
\end{aligned}
$$

Let $x=x_{i}$ in (1)

$$
\frac{\partial u\left(x_{i}, t\right)}{\partial t}-a(t) \frac{\partial^{3} u\left(x_{i}, t\right)}{\partial t \partial x^{2}}=b(t) \frac{\partial^{2} u\left(x_{i}, t\right)}{\partial x^{2}}+c(t) u\left(x_{i}, t\right)+d(t) u\left(x_{i}, t-r\right)+f\left(x_{i}, t\right), x_{i} \in \omega_{h}, t \in(0, T]
$$

Using formula (4) in (5), we obtain

$$
\frac{1}{12}\left[u_{i+1}^{\prime}(t)+10 u_{i}^{\prime}(t)+u_{i-1}^{\prime}(t)\right]-a(t) u_{\bar{x} x, i}^{\prime}(t)=b(t) u_{\bar{x} x, i}(t)
$$


$+\frac{c(t)}{12}\left[u_{i+1}(t)+10 u_{i}(t)+u_{i-1}(t)\right]+\frac{d(t)}{12}\left[u_{i+1}(t-r)+10 u_{i}(t-r)+u_{i-1}(t-r)\right]$

$+\tilde{f}_{i}(t)+R_{i}(t), i=1,2, \ldots N-1$,

$u_{i}(t)=\varphi_{i}(t)$,

$u_{0}(t)=u_{N}(t)=0$,

where

$\tilde{f}_{i}(t)=\frac{1}{12}\left[f_{i+1}(t)+10 f_{i}(t)+f_{i-1}(t)\right]$,
$R_{i}(t)=a(t) \frac{h^{4}}{240} \frac{\partial^{7} u\left(\xi_{i}, t\right)}{\partial t \partial x^{6}}+b(t) \frac{h^{4}}{240} \frac{\partial^{6} u\left(\xi_{i}, t\right)}{\partial x^{6}}, \xi_{i} \in\left(x_{i-1}, x_{i+1}\right)$.

Taking into account the following relations

$\frac{1}{12}\left[u_{i+1}^{\prime}(t)+10 u_{i}^{\prime}(t)+u_{i-1}^{\prime}(t)\right]=u_{i}^{\prime}(t)+\frac{h^{2}}{12} u_{\bar{x}, i}^{\prime}(t)$,

$\frac{c(t)}{12}\left[u_{i+1}(t)+10 u_{i}(t)+u_{i-1}(t)\right]=c(t) u_{i}(t)+\frac{h^{2}}{12} c(t) u_{\bar{x} x, i}(t)$,

$\frac{d(t)}{12}\left[u_{i+1}(t-r)+10 u_{i}(t-r)+u_{i-1}(t-r)\right]=d(t) u_{i}(t-r)+\frac{h^{2}}{12} d(t) u_{\bar{x} x, i}(t-r)$

and neglecting the remainder term $R_{i}$ in (6), we propose the following differentialdifference scheme

$$
\begin{aligned}
& y_{i}^{\prime}(t)-\left(a(t)-\frac{h^{2}}{12}\right) y_{\bar{x} x, i}^{\prime}(t)=\left(b(t)+c(t) \frac{h^{2}}{12}\right) y_{\bar{x} x, i}(t)+c(t) y_{i}(t)+d(t) y_{i}(t-r) \\
& +d(t) \frac{h^{2}}{12} y_{\bar{x} x, i}(t-r)+\tilde{f}_{i}(t), i=1,2, \ldots, N-1, t \in(0, T], \\
& y_{i}(t)=\varphi_{i}(t), i=0,1, \ldots, N, t \in(0, T] \text {, } \\
& y_{0}(t)=y_{N}(t)=0, t \in(0, T] \text {. }
\end{aligned}
$$

For the error function $z_{i}(t)=y_{i}(t)-u_{i}(t)$, from the relations (6)-(8) and (9)-(11), we have the following differential-difference problem 


$$
\begin{aligned}
& \begin{aligned}
z_{i}^{\prime}(t)-\left(a(t)-\frac{h^{2}}{12}\right) z_{\bar{x} x, i}^{\prime}(t) & =\left(b(t)+c(t) \frac{h^{2}}{12}\right) z_{\bar{x} x, i}(t)+c(t) z_{i}(t)+d(t) z_{i}(t-r) \\
& +d(t) \frac{h^{2}}{12} z_{\bar{x} x, i}(t-r)-R_{i}(t), i=1,2, \ldots, N-1
\end{aligned} \\
& z_{i}(t)=0, t \in(0, T], \\
& z_{0}(t)=z_{N}(t)=0, t \in(0, T] .
\end{aligned}
$$

\section{A PRIORI ESTIMATE}

In this section, we give a lemma which is used in the next section for establishing the error estimate

Lemma 3.1. Let $a, b, f \in C[0, T]$ and $\varphi \in C[-r, 0]$. Then the solution of the following initial value problem

$v^{\prime}(t)+a(t) v(t)+b(t) v(t-r)=f(t), 0<t \leq T$,

$v(t)=\varphi(t),-r \leq t \leq 0$,

provides the following inequality

$$
|v(t)| \leq\left(c_{0}+c_{1} \int_{-r}^{0}|\varphi(\eta)| d \eta\right) e^{c_{1} t}, 0 \leq t \leq T
$$

Here

$$
\begin{aligned}
& c_{0}=\left(|\varphi(0)|+\|f\|_{1}\right) \max \left\{1, e^{-a_{*} T}\right\}, \\
& c_{1}=\|b\|_{\infty} \max \left\{1, e^{-a_{*} T}\right\}, \\
& a_{*}=\min _{[0, T]} a(t), \\
& \|f\|_{1}=\int_{0}^{T}|f(t)| d t, \\
& \|b\|_{\infty}=\max _{[0, T]}|b(t)| .
\end{aligned}
$$

Proof. For the solution of (15)-(16), we can write

$$
v(t)=v(0) e^{-\int_{0}^{t} a(\eta) d \eta}-\int_{0}^{t} b(\tau) v(\tau-r) e^{-\int_{\tau}^{t} a(\eta) d \eta} d \tau+\int_{0}^{t} f(\tau) e^{-\int_{\tau}^{t} a(\eta) d \eta} d \tau .
$$

From this relation, we get 
$|v(t)| \leq\left(|\varphi(0)|+\|f\|_{1}\right) \max \left\{1, e^{-a_{*} T}\right\}+\|b\|_{\infty} \max \left\{1, e^{-a_{*} T}\right\} \int_{0}^{t}|v(\tau-r)| d \tau$.

After denoting $\delta(t)=|v(t)|$, the inequality (18) reduces to

$\delta(t) \leq c_{0}+c_{1} \int_{0}^{t} \delta(\tau-r) d \tau$

Using variable transformation $\tau-r=\eta$ in (19), it can be seen clearly that

$\delta(t) \leq c_{0}+c_{1} \int_{-r}^{t-r} \delta(\eta) d \eta \leq c_{0}+c_{1} \int_{-r}^{0}|\varphi(\eta)| d \eta$ for $0<t \leq r$,

$\delta(t) \leq c_{0}+c_{1} \int_{-r}^{0}|\varphi(\eta)| d \eta+c_{1} \int_{0}^{t-r} \delta(\eta) d \eta$ for $t>r$.

From here, by virtue of Gronwall's inequality, we easily arrive at (17).

\section{THE ERROR ESTIMATE}

Now we give the main result of this paper.

Theorem 4.1. Let the derivatives $\frac{\partial^{7} u}{\partial t \partial x^{6}}, \frac{\partial^{6} u}{\partial x^{6}}$ are bounded on the $\bar{Q}$ and $\alpha-\frac{h^{2}}{12} \geq \alpha_{*}>0$.

Then the error of the problem (9)-(11) satisfies

$\left|y_{i}(t)-u_{i}(t)\right| \leq C h^{4}, i=0,1, \ldots, N, t \in(0, T]$,

where $C$ is a constant which is independent of $h$.

Proof. Let $Z(t)=\left(z_{1}(t), z_{2}(t), \ldots, z_{N-1}(t)\right)^{T}$. Then the scheme (12)-(14) can be expressed in vector form as

$$
\begin{aligned}
& Z^{\prime}(t)+\left(a(t)-\frac{h^{2}}{12}\right) M Z^{\prime}(t)=-\left(b(t)+\frac{c(t) h^{2}}{12}\right) M Z(t)+c(t) Z(t)+d(t) Z(t-r) \\
& -\frac{d(t) h^{2}}{12} M Z(t-r)+\mathfrak{R}(t), \\
& Z(0)=\mathbf{0},
\end{aligned}
$$

where 


$$
\begin{aligned}
& \mathfrak{R}(t)=\left(R_{1}(t), R_{2}(t), \ldots, R_{N-1}(t)\right)^{T},
\end{aligned}
$$

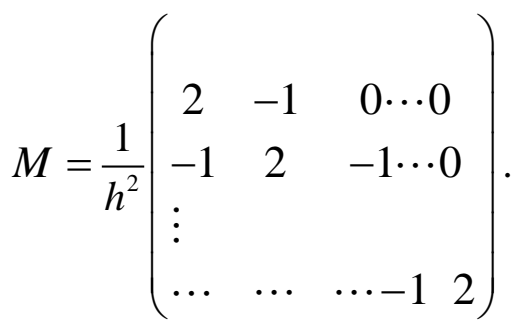

The matrix $M$ can be diagonalized as $[14,16]$

$$
M=B^{-1} \text { diagonal }\left(\lambda_{1}, \lambda_{2}, \ldots \lambda_{N-1}\right) B,
$$

with

$$
\begin{aligned}
& B=B^{-1}=\left(b_{i k}\right)_{i, k=1}^{N-1}=\left((-1)^{i+k} \sqrt{\frac{2}{N}} \sin \frac{\pi i k}{N}\right)_{i, k=1}^{N-1}, \\
& \lambda_{i}=\frac{4}{h^{2}} \cos ^{2}\left(\frac{\pi i}{2 N}\right), i=1, \ldots, N-1 .
\end{aligned}
$$

Multiplying equation (21) on the left by $B$ and denoting

$$
\begin{aligned}
& B Z(t)=\Psi(t)=\left(\psi_{1}(t), \psi_{2}(t), \ldots, \psi_{N-1}(t)\right)^{T}, \\
& B \mathfrak{R}(t)=\Phi(t)=\left(\phi_{1}(t), \phi_{2}(t), \ldots, \phi_{N-1}(t)\right)^{T},
\end{aligned}
$$

the initial-value problem (21)-(22) is turned into the decomposed system as :

$$
\begin{aligned}
& \psi_{s}^{\prime}(t)+\left(a(t)-\frac{h^{2}}{12}\right) \lambda_{s} \psi_{s}^{\prime}(t)=-\left(b(t)+\frac{c(t) h^{2}}{12}\right) \lambda_{s} \psi_{s}(t)+c(t) \psi_{s}(t) \\
& +d(t) \psi_{s}(t-r)-\frac{d(t) h^{2}}{12} \lambda_{s} \psi_{s}(t-r)+\phi_{s}(t), s=1,2, \ldots, N-1, \\
& \psi_{s}(0)=0, s=1,2, \ldots, N-1 .
\end{aligned}
$$

If we rewrite (23) in the form

$$
\psi_{s}^{\prime}(t)+\frac{\left(b(t)+\frac{c(t) h^{2}}{12}\right) \lambda_{s}-c(t)}{1+\lambda_{s}\left(a(t)-\frac{h^{2}}{12}\right)} \psi_{s}(t)+\frac{\frac{d(t) h^{2}}{12} \lambda_{s}-d(t)}{1+\lambda_{s}\left(a(t)-\frac{h^{2}}{12}\right)} \psi_{s}(t-r)=\frac{\phi_{s}(t)}{1+\lambda_{s}\left(a(t)-\frac{h^{2}}{12}\right)}
$$

with 


$$
A_{s}(t)=\frac{\left(b(t)+\frac{c(t) h^{2}}{12}\right) \lambda_{s}-c(t)}{1+\lambda_{s}\left(a(t)-\frac{h^{2}}{12}\right)}, \quad B_{s}(t)=\frac{\frac{d(t) h^{2}}{12} \lambda_{s}-d(t)}{1+\lambda_{s}\left(a(t)-\frac{h^{2}}{12}\right)}
$$

and after then we apply Lemma 3.1, we conclude

$$
\left|\psi_{s}(t)\right| \leq\left\|\frac{\phi_{S}}{1+\lambda_{s}\left(a-\frac{h^{2}}{12}\right)}\right\|_{1} \max \left\{1, e^{-A_{*} T}\right\} e^{c_{1, s} t},
$$

where

$$
A_{*}=\min _{[0, T]} A_{s}(t), \quad c_{1, s}=\left\|B_{s}\right\|_{\infty} \max \left\{1, e^{-A_{*} T}\right\} .
$$

$A_{*}$ and $c_{1, s}$ are uniformly bounded independently of $h$.

For $A_{s}$, it can be written that

$$
\left|A_{s}(t)\right| \leq\left|\frac{\left(b(t)+\frac{c(t) h^{2}}{12}\right) \lambda_{s}}{1+\lambda_{s}\left(a(t)-\frac{h^{2}}{12}\right)}\right|+\left|\frac{c(t)}{1+\lambda_{s}\left(a(t)-\frac{h^{2}}{12}\right)}\right| .
$$

Also as $\alpha-\frac{h^{2}}{12} \geq \alpha_{*}>0$ and $\lambda_{s}$ constants are bounded below with $\lambda_{1}$, it follows that

$$
1+\lambda_{s}\left(a(t)-\frac{h^{2}}{12}\right) \geq \alpha_{0}>0 .
$$

Taking then into account (27) in (26), we get

$$
\begin{aligned}
\left|A_{s}(t)\right| & \leq \frac{\left(\|b\|_{\infty}+\frac{\|c\|_{\infty} h^{2}}{12}\right) \lambda_{s}}{1+\lambda_{s} \alpha_{*}}+\alpha_{0}^{-1}\|c\|_{\infty} \\
& \leq \alpha_{*}^{-1}\left(\|b\|_{\infty}+\frac{\|c\|_{\infty} h^{2}}{12}\right)+\alpha_{0}^{-1}\|c\|_{\infty} .
\end{aligned}
$$

If we pay attention to $h \leq \frac{l}{2}$, then it follows that $\left|A_{s}(t)\right| \leq \alpha_{*}^{-1}\left(\|b\|_{\infty}+\frac{\|c\|_{\infty} l^{2}}{48}\right)+\alpha_{0}^{-1}\|c\|_{\infty}$.

In the similar way, for $B_{s}$ we obtain 
$\left|B_{s}(t)\right| \leq \alpha_{*}^{-1}\|d\|_{\infty} \frac{l^{2}}{48}+\alpha_{0}^{-1}\|c\|_{\infty}$.

Thereby the constants $A_{*}$ and $c_{1, s}$ are bounded independently of $h$. Therefore the inequality (25) yields

$\left|\psi_{s}(t)\right| \leq C \lambda_{s}^{-1} \alpha_{*}^{-1}\left\|\phi_{s}\right\|_{1}$.

Since

$\left|\phi_{s}(t)\right| \leq \sum_{k=1}^{N}\left|b_{s k}\right|\left|R_{k}\right| \leq \sqrt{\frac{2}{N}} \sum_{k=1}^{N}\left|R_{k}\right| \leq \sqrt{\frac{2}{N}}(N-1) C h^{4} \leq C \sqrt{N} h^{4} \leq C h^{3.5}$,

the inequality (28) leads to

$\left|\psi_{s}(t)\right| \leq \alpha_{*}^{-1} \lambda_{s}^{-1} C h^{3.5}$.

Further, from the relation

$z_{i}(t)=\sum_{k=1}^{N-1} b_{i k} \psi_{k}$

we then obtain

$$
\left|z_{i}(t)\right| \leq \alpha_{*}^{-1} C h^{3.5} \sum_{k=1}^{N-1} \lambda_{k}^{-1}\left|b_{i k}\right| \leq C h^{3.5} \sqrt{\frac{2}{N}}(N-1) \sum_{k=1}^{N-1} \frac{h^{2}}{4 \cos ^{2}\left(\frac{\pi k}{2 N}\right)} \leq C h^{4} h^{2} \sum_{k=1}^{N-1} \frac{1}{4 \sin ^{2}\left(\frac{\pi(N-k)}{2 N}\right)} .
$$

Taking into account the following inequality

$\sin x>\frac{2}{\pi} x, 0<x<\frac{\pi}{2}$,

in (4.10), consequently we obtain

$\left|z_{i}(t)\right| \leq C h^{6} \sum_{k=1}^{N-1} \frac{1}{\frac{4}{\pi^{2}}\left(\frac{\pi(N-k)}{2 N}\right)^{2}}=C h^{6} N^{2} \sum_{k=1}^{N-1} \frac{1}{(N-k)^{2}} \leq C h^{4}$.

\section{NUMERICAL EXAMPLE AND CONCLUSION}

Consider the problem

$$
\begin{aligned}
& \frac{\partial u}{\partial t}-\frac{\partial^{3} u}{\partial t \partial x^{2}}=\frac{\partial^{2} u}{\partial x^{2}}+t^{2} u(x, t-1)-\sin (\pi x)\left(e^{-t}+t^{2}\right),(x, t) \in[0,1] \times(0,2], \\
& u(x, t)=\sin (\pi x),(x, t) \in[0,1] \times[-1,0] \\
& u(0, t)=u(1, t)=0, t \in(0,2] .
\end{aligned}
$$

The exact solution of this problem is 
$u(x, t)=\left\{\begin{array}{l}e^{-t} \sin (\pi x), \quad \mathrm{t} \in(0,1] \\ \left(e^{\frac{t+\pi^{2}}{1+\pi^{2}}}\left(2 \pi^{6}+6 \pi^{4}+5 \pi^{2}+1\right) / \pi^{2}-\left(\left(2 \pi^{6}+6 \pi^{4}+6 \pi^{2}+2\right) e-\pi^{2}\right) /\left(1+\pi^{2}\right)\right. \\ \left.+\left(e^{t}\left(1+1 / \pi^{2}\right)+2 t e\left(\pi^{2}+1\right)^{2}+t^{2} e\left(\pi^{2}+1\right)-1\right) /\left(1+\pi^{2}\right)\right) e^{-t} \sin (\pi x), \quad \mathrm{t} \in(1,2]\end{array}\right.$

To solve this problem numerically, we use the appropriate Runge-Kutta method. The spatial and time steps are both taken to be 0.1 .The values for exact and numerical solutions and appropriate pointwise errors are shown in Table 1 and Table 2.

Table 1 . The results on $[0,1] \times[0,1]$

\begin{tabular}{|l|l|l|l|}
\hline$(x, t)$ & Exact Solution & R.K. Approximation & Pointwise Error \\
\hline$(0.1,0.1)$ & 0.2796101393 & 0.27965712282 & $4.698352 \times 10^{-5}$ \\
\hline$(0.2,0.2)$ & 0.4812378623 & 0.48132723027 & $8.936797 \times 10^{-5}$ \\
\hline$(0.3,0.3)$ & 0.5993345303 & 0.5994575349 & $1.230046 \times 10^{-5}$ \\
\hline$(0.4,0.4)$ & 0.6375122478 & 0.6376568482 & $1.446004 \times 10^{-4}$ \\
\hline$(0.5,0.5)$ & 0.6065306597 & 0.6066827016 & $1.520419 \times 10^{-4}$ \\
\hline$(0.6,0.6)$ & 0.5219508827 & 0.5220954831 & $1.446004 \times 10^{-4}$ \\
\hline$(0.7,0.7)$ & 0.4017459499 & 0.4018689545 & $1.230046 \times 10^{-4}$ \\
\hline$(0.8,0.8)$ & 0.2641089385 & 0.26419830647 & $8.936797 \times 10^{-5}$ \\
\hline$(0.9,0.9)$ & 0.1256369343 & 0.12568391782 & $4.698352 \times 10^{-5}$ \\
\hline
\end{tabular}

Table 2. The results on $[0,1] \times[1,2]$

\begin{tabular}{|l|l|l|l|}
\hline$(x, t)$ & Exact Solution & R.K. Approximation & Pointwise Error \\
\hline$(0.1,1.1)$ & 16.4311515638 & 16.4312156615 & $6.40977 \times 10^{-5}$ \\
\hline$(0.2,1.2)$ & 28.540363147 & 28.5404850681 & $1.219211 \times 10^{-4}$ \\
\hline$(0.3,1.3)$ & 35.872352318 & 35.872520128 & $1.678100 \times 10^{-4}$ \\
\hline$(0.4,1.4)$ & 38.5102577648 & 38.5104550372 & $1.972724 \times 10^{-4}$ \\
\hline$(0.5,1.5)$ & 36.9781050461 & 36.9783124708 & $2.074245 \times 10^{-4}$ \\
\hline$(0.6,1.6)$ & 32.1168015304 & 32.1169988028 & $1.972724 \times 10^{-4}$ \\
\hline$(0.7,1.7)$ & 24.9500651436 & 24.9502329536 & $1.678100 \times 10^{-4}$ \\
\hline$(0.8,1.8)$ & 16.5549350971 & 16.5550570182 & $1.219211 \times 10^{-4}$ \\
\hline$(0.9,1.9)$ & 7.9486324554 & 7.9486965531 & $6.409770 \times 10^{-5}$ \\
\hline
\end{tabular}

It can be concluded that numerical results are consistent with the theoretical results.

\section{REFERENCES}

1. G. M. Amiraliyev, Difference method for the solution of one problem of the theory of dispersive waves, USSR Differential Equations 26, 2146-2154, 1990.

2. G. M. Amiraliyev and Y. D. Mamedov, Difference schemes on the uniform mesh for a singularly perturbed pseudo-parabolic equations, Turkish Journal of Mathematics 19, 207-222, 1995. 
3. G. M. Amiraliyev and I. G. Amireliyeva, Difference schemes for the singularly perturbed Sobolev equations, in Proceedings of the International Conference on Difference Equations, Special Functions and Applications, Munich, 23-40, 2005.

4. G. M. Amiraliyev, H. Duru and I. G. Amireliyeva, A parameter-uniform numerical method for a Sobolev problem with initial layer, Numerical Algorithms 44, 185-203, 2007.

5. G. Barenblatt, I. Zheltov and I. Kochina, Basic concepts in the theory of seepage of homogeneous liquids in fissured rocks, Journal of Applied Mathematics and Mechanics 24, 1286-1303, 1960.

6. A. Bouzani, Initial-boundary value problems for a class of pseudoparabolic equations with integral boundary conditions, Journal of Mathematical Analysis and Appications 291, 371-386, 2004.

7. P. J. Chen and M. E. Gurtin, On a theory of heat conduction involving two temperatures, Zeitschrift für angewandte Mathematik und Physik 19, 614-627, 1968.

8. P. L. Davis, A quasilinear parabolic and related third order problem, Journal of Mathematical Analysis and Applications 49, 327-335, 1970.

9. R. E. Ewing, Time-stepping Galerkin methods for nonlinear Sobolev partial differential equations, SIAM Journal on Numerical Analysis 15, 1125-1150, 1978.

10. W. H. Ford and T. W. Ting, Uniform error estimates for difference approximations to nonlinear pseudo-parabolic partial differential equations, SIAM. Journal on Numerical Analysis 15, 155-169, 1974.

11. H. Gu, Characteristic finite element methods for nonlinear Sobolev equations, Applied Mathematics and Computation 102, 51-62, 1999.

12. R. Huilgol, A second order fluids of the differential type, International Journal of Non-Linear Mechanics 3, 471-482, 1968.

13. J.H. Lightbourne and S. M. Rankin, A partial functional differential equation of Sobolev type, Journal of Mathematical Analysis and Applications 93, 328-337, 1983.

14. S. B. Nemchinov, On the finite difference method to the elliptic boundary value problems, Journal of Compuational Mathematics and Mathematical Physics 2, 418436, 1962. (in Russian)

15. E. Reyes, F. Rodriguez and J. A. Martin, Analytic-numerical solutions of diffusion mathematical models with delays, Journal of Computational and Applied Mathematics 56, 743-753, 2008.

16. A. A. Samarskii, The Theory of Difference Schemes, Marcel Dekker, New York, 2001.

17. T. Sun and D. Yang, The finite difference streamline diffusion methods for Sobolev equations with convection-dominated term, Applied Mathematics and Computation 125, 325-345, 2002.

18. H. Tian, Asymptotic stability of numerical method for linear delay parabolic differential equations, Journal of Computational and Applied Mathematics 56, 1758$1765,2008$.

19. T. W. Ting, Certain non-steady flows of second-order fluids, Archive for Rational Mechanics and Analysis 14, 1-26, 1963.

20. M. Yang, Analysis of second order finite volume element methods for pseudoparabolic equations in three spatial dimensions, Applied Mathematics and Computation 196, 94-104, 2008. 